Vol. 4. No. 2. (221-239). Julio-Dic/2019

ISSN: 2539-1518

\title{
Corrientes de pensamiento en la educación ambiental y ámbitos de aplicación
}

\author{
Galo Ladino Nuñez Aldaz \\ glnunez@uce.edu.ec \\ Universidad Central del Ecuador \\ María Cristina García \\ garciabellizzi@gmail.com \\ Universidad Central del Ecuador
}

\section{Resumen}

El propósito del presente trabajo fue analizar la aplicabilidad de varias corrientes teóricas en educación ambiental con especial atención en el manejo de los recursos naturales del Ecuador atendiendo a la urgencia de crear mecanismos e instrumentos que contribuyan con el desarrollo sostenible del planeta, en razón de que la sociedad ecuatoriana clama por el mejoramiento ambiental y de su calidad de vida. La metodología seguida para el desarrollo de la investigación tuvo un enfoque cualitativo, en tal sentido se llevó a cabo un análisis documental, se utilizaron los métodos teóricos y empíricos, como inductivo-deductivo, analítico-sintético, su alcance es descriptivo, se abordan los principales aspectos teóricos de las corrientes ambientalistas. Como resultados se recogen datos que evidencian la necesidad de la educación ambiental para el cambio de comportamiento y lograr la concienciación para el cuidado ambiental.

\section{Palabras clave}

corrientes ambientalistas, educación ambiental, desarrollo sostenible.

Recibido 02/09/2019 - 28/10/2019. 


\section{Introducción}

Desde su legitimación como campo pedagógico, la educación ambiental (EA) se ha encontrado en un permanente antagonismo, o al menos divergencia, de enfoques y posturas teóricas y metodológicas. González (2006) plantea que la EA ha arropado numerosos discursos, desde sus vínculos con la enseñanza de la ecología (como una rama de las ciencias naturales) y la educación para la conservación hasta los enfoques con fuertes cargas en la dimensión cívica, en la formación ciudadana, la educación moral y ética; la dimensión política, desde el análisis crítico de la globalización, la inequidad social y la relación norte-sur, así como la dimensión rural.

Asimismo, en nombre de la EA se ha justificado una pluralidad enorme de discursos que simultáneamente han apuntalado y minado una percepción social favorable de su campo, por lo que muchos de los discursos identificados en la EA se encuentran fuertemente asociados a un modo de concebir la realidad que busca la verdad universal y cree en el camino de plenitud y progreso, en el proyecto de la humanidad, en la inviolabilidad del sujeto humano y en una concepción de un mundo social e histórico ordenado por fundamentos absolutos, entre otros rasgos.

En este sentido la EA ha sido un punto de confluencia de especialistas y practicantes no sólo provenientes de las ciencias sociales y humanas, así como de las naturales y exactas, sino de las más disímbolas actividades y creencias. Sin embargo, en esta compleja trayectoria ha sido evidente la intención de hegemonizar el campo de la EA por parte de aquellas aproximaciones ligadas al ambiente con la mirada puesta en la escolarización y en la racionalidad instrumental, por encima de cualquiera de las corrientes del pensamiento comprometidas con los complejos problemas socio ambientales de nuestro tiempo.

El propio Programa Internacional de Educación Ambiental (UNESCO-PNUMA, 1975-1995) contribuyó de manera intensiva a esta pretensión, cuya serie de publicaciones valoró y dio voz a las experiencias y perspectivas de representantes de los países centrales. En la conferencia de las Naciones Unidas celebrada en Rio de Janeiro sobre el medio ambiente y desarrollo sostenible en una de sus declaraciones dice "Procurando alcanzar 
acuerdos internacionales en los que se respeten los intereses de todos y se proteja la integridad del sistema ambiental y de desarrollo mundial".

La población mundial tenía la mirada puesta en estos encuentros mundiales para salvar lo poco que queda de espacios no contaminados; salvar para dejar un legado a las futura generaciones, pero solo ha quedado en discursos para campañas publicitarias como justificación de las reuniones internacionales sobre el cuidado ambiental, porque los países comprometidos, especialmente los llamados desarrollados no tuvieron la voluntad política y financiera para contrarrestar la extracción a gran escala a nivel mundial. Se hace evidente que los acuerdos establecidos en la Conferencia de Estocolmo, y la de Johannesburgo no tienen logros importantes en la solución a la crisis ambiental con repercusiones sociales a nivel mundial; los recursos de los países pobres han sido saqueados y alejados del desarrollo equitativo.

La industrialización de los recursos naturales, al ser procesados tecnología no amigable con el medio ambiente, ha provocado un desarrollo económico desmedido, dejando de lado acuerdos internacionales sobre sustentabilidad, que consagra la equidad social y garantiza al medio ambiente la biodiversidad ecológica de manera responsable, dando seguridad de vida a todas las especies, incluyendo la humana, con las mismas oportunidades de crecimiento y progreso social.

En este orden de ideas, la Carta de Belgrado en octubre de 1975 plantea que "es absolutamente vital que todos los ciudadanos del mundo insistan en medidas que apoyen un tipo de crecimiento económico que no tenga repercusiones perjudiciales para las personas, para su ambiente ni para sus condiciones de vida”. Estas declaraciones solo han quedado en retórica, pues los países desarrollados han saqueado los recursos naturales del mundo sin importarles la vida de la naturaleza ni de la humanidad, poniendo por delante como prioridad el desarrollo económico desmedido.

En este sentido se evidencia una delimitación de obligaciones de acuerdos mundiales, donde no se responsabiliza de los desembolsos económicos para invertir en educación ambiental; así mismo la falta de recursos afecta el avance de programas para mejorar y elevar la conciencia ambiental, en especial de los grandes inversionistas dedicados a la extracción de recursos naturales. En este punto contrastan pensadores y corrientes ambientalista, quienes manifiestan que, al momento de solucionar los 
problemas ambientales, prevalecen los intereses económicos limitando la recuperación del medio ambiente y la contaminación ambiental. A pesar de estas limitaciones y ante la crisis en el marco político -institucional de los países Iberoamericanos, se acordaron estrategias educativas, como la "Declaración de Cochabamba y las recomendaciones sobre Políticas Educativas al inicio del siglo XXI" (UNESCO 2001), donde los países participantes plantearon a la educación ambiental como prioridad para el desarrollo social sustentable y conciencia ambiental, en todos los niveles del sistema educativo.

En el Ecuador, los intereses económicos han prevalecido en las transnacionales encargadas de extraer los recursos del ambiente, sin importar la biodiversidad del territorio ecuatoriano, por lo que las actividades petroleras se han convertido en amenaza permanente de contaminación del entorno natural del oriente amazónico ecuatoriano, desplazado la flora y fauna de la región, así como a los nativos de la amazonia. A esto le debemos añadir la explotación minera, deforestación de los bosques originarios, afectando los ríos del Ecuador con un alto grado de contaminación, trayendo como consecuencia que muchos de ellos no sean aptos para consumo humano, así como para las otras especies vivientes de la región.

Un ejemplo de ello es la inclusión de aspectos medioambientales en la Constitución y el Ministerio del Ambiente, entre los cuales contemplan la protección del medio ambiente; sin embargo, poco se ha logrado en cuanto a generar conciencia sobre su preservación, muy por el contario sigue prevaleciendo el interés de lo económico, dejando en el plano secundario la equidad social. Adicionalmente existen Organizaciones no gubernamentales (ONG) y Fundaciones dedicadas a la lucha por la conservación y protección de la naturaleza; a ellas se suman los indígenas y campesinos que denuncian permanentemente el abuso, el atropello de las petroleras transnacionales, que prefieren enfrentar las demandas judiciales en vez de atender y reparar los daños causados a la naturaleza, la biodiversidad, a la integridad de las comunidades indígenas y colonos que habitan esos territorios.

A partir del momento en el que se aprueba en Ecuador el Plan Decenal de Educación en 2006 se han realizado reformas al Sistema Educativo, atendiendo lo contemplado en la nueva Ley de Educación aprobada por la Asamblea Nacional; sin embargo estas ni responden a una clara política estatal, ni a lo consagrado en la Constitución de la República del Ecuador, que manifiesta el Buen Vivir con el cuidado 
de la Pacha mama, devolviendo a la naturaleza la biodiversidad en su estado natural y los recursos naturales para el desarrollo integral de sus pueblos. No obstante, hay que reconocer que la EA ha reposicionado los procesos educativos, en el marco de las políticas internacionales y nacionales, en un álgido momento en el que los asuntos ambientales han perdido progresivamente vigor y actualidad

\section{Objetivo de la investigación}

Analizar la aplicabilidad de las corrientes teóricas de la educación ambiental con especial atención en el manejo de los recursos naturales para el desarrollo sostenible en el Ecuador

\section{Desarrollo}

La Educación Ambiental (EA) forma parte de una larga trayectoria histórica, a través de la cual ha ido adquiriendo pertinencia desde el punto de vista social, ambiental, económico y educativo. Cuando se aborda el campo de la EA, se observa que, a pesar de existir una preocupación común por el medio ambiente y el reconocimiento del papel central de la educación para el mejoramiento de la relación con este último, se adoptan diferentes discursos sobre la EA y proponen diversas maneras de concebir y de practicar la acción educativa en este campo.

Debido a estas proyecciones, las exigencias, según manifiesta Avendaño (2012), en la revista "Luna Azul" en torno al tema ambiental y a su protección han originado diversas tendencias e instrumentos, los cuales buscan mitigar los efectos producidos por el uso irracional de los recursos, la tecnificación. No ha sido suficiente los esfuerzos para mitigar la protección ambiental, hace falta voluntad y decisiones políticas y económicas.

Según la Organización de las Naciones Unidas ONU en la conferencia de Estocolmo sobre el Medio Ambiente Humano, su recomendación fue, un mayor desarrollo de la Educación Ambiental, para enfrentar seriamente la crisis ambiental del mundo. Dirigieron la mirada a la educación ambiental, pero los recursos económicos fueron los limitantes para lograr las metas propuestas de elevar la conciencia ambiental.

La "Cumbre Mundial sobre el Desarrollo Sostenible" (2002) celebrada el 26 de agosto al 4 de septiembre de 2002 en Johannesburgo (África del Sur), fue una cumbre mundial del desarrollo sostenible organizada por la ONU, encargada de brindar apoyo a las personas sin dañar el medio ambiente. Podemos deducir que este apoyo no se ha 
cumplido, la devastación del medio ambiente empeora cada vez con mayor fuerza por las empresas del monopolio internacional, solo le interesa la ambición del dinero, nada por la vida de la naturaleza y la humanidad.

La EA es un proceso de formación que busca despertar la conciencia ambiental y que las personas se identifiquen con los graves problemas ambientales (Cugler, 2010) por los que se encuentran pasando las sociedades y el planeta. Desde la perspectiva de Copello, Nunes, Langoni y Russo la EA “consiste en un modo de ver el mundo, que evidencia las interrelaciones y las interdependencias de los diversos elementos que constituyen y mantienen la vida" (2008, p. 44). Para ello se ha buscado emplear estrategias desde diversos enfoques, las cuales tienen una gran multiplicidad de manifestaciones (González y Valdez, 2012) haciendo de la EA una dimensión compleja.

La sistematización de las corrientes de pensamiento en educación ambiental, resultan una herramienta de análisis al servicio de la indagación de la diversidad de proposiciones pedagógicas y no un subterfugio que obliga a clasificar todo en categorías rígidas, con el riesgo de deformar la realidad. Es así como a lo largo del trabajo se hace un recorrido de las corrientes de la educación ambiental. Algunas tienen una tradición más antigua y han sido dominantes en las primeras décadas de la EA (los años 1970 y 1980), como: la corriente naturalista; la corriente conservacionista; la corriente resolutiva; la corriente sistémica; la corriente científica; la corriente humanista; la corriente moral/ética. Otras corresponden a preocupaciones que han surgido recientemente, como: la corriente holística; la corriente bio-regionalista; la corriente práxica; la corriente crítica; la corriente etnográfica; la corriente de eco-educación, y la corriente de la sostenibilidad/sustentabilidad.

\section{Corrientes de pensamiento en la educación ambiental (EA)}

La diversidad de corrientes teóricas y prácticas en educación ambiental muestra a la vez un pluralismo de concepciones y de enfoques coexistentes, resultado del enriquecimiento progresivo de este campo y de una evolución hacia una captación más radical de las dimensiones ética, cultural y política de las cuestiones ambientales, tal como se desarrolla a continuación: 


\section{Corrientes de larga tradición}

Esta corriente corresponde a la década de los setenta y ochenta. Dentro de ella se conocen: a) corriente naturalista, b) corriente conservacionista/recursista, c) corriente resolutiva, d) corriente sistémica, e) corriente científica, f) corriente humanista y g) corriente moral/ética.

\section{a.- Corriente Naturalista}

La corriente naturalista es muy antigua, consideran el aprendizaje por inmersión e imitación en los grupos sociales que está estrechamente forjada en la relación con el medio natural. En el transcurro del siglo pasado, la corriente naturalista se vincula con la educación al medio natural y al aire libre. Para Sauvé (2005) esta corriente está centrada en la relación con la naturaleza. El enfoque educativo puede ser cognitivo (aprender de las cosas sobre la naturaleza) o experiencial (vivir en la naturaleza y aprender de ella) o afectivo, o espiritual o artístico (asociando la creatividad humana a la de la naturaleza).

Como se puede analizar para la corriente naturalista, realizar aprendizajes sobre educación ambiental en un ambiente natural es el momento y el lugar apropiado para generar en el estudiantado la mejor forma de comprender la importancia de conservar un ambiente natural en condiciones saludables que permiten el desarrollo de la vida en sus diversas manifestaciones, respetando los espacios de los seres vivos.

Se puede inferir lo que dice este pensador, nada mejor y elevar la conciencia ambiental cuando aprendemos en contacto con la naturaleza en un acercamiento directo, que permite tener vínculos entre el conocimiento teórico con la practico ambiental de una manera amigable.

\section{b.- Corriente conservacionista / recursista}

Sus defensores con biólogos y ecólogos, quienes ponían límites físicos al crecimiento económico y poblacional, y el crecimiento cero. Esta corriente, según Corcuera y Ponce de León (2004) agrupa las proposiciones centradas en la conservación de los recursos, tanto en lo que concierne a su calidad como a su cantidad: el agua, el suelo, la energía, las plantas (principalmente las plantas comestibles y medicinales) y los animales (por los recursos que se pueden obtener de ellos), el patrimonio genético, el patrimonio construido, etc. Cuando se habla de conservación de la naturaleza, como de la 
biodiversidad, se trata sobre todo de una naturaleza-recurso. Se evidencia aquí una preocupación por la gestión del medio ambiente, llamada más bien gestión. A partir de allí se expone, como posible salida a la presión ambiental y el consecuente riesgo para la vida, el crecimiento cero o el estado estacionario. El primero que abogaba por el freno al crecimiento demográfico, y el segundo, por no producir por encima del crecimiento de la población y sus necesidades, por cuanto existe dependencia de la naturaleza para la satisfacción de esas necesidades y los tiempos de recurrencia (de las necesidades humanas) son más cortos que los de reproducción de la naturaleza (Sánchez y Aguilera 2014).

Aquí se puede deducir que existe una exageración en sus criterios, ya que el desarrollo social es dialectico y no podemos poner límites, al contrario, y estamos de acuerdo con esta corriente es que debe haber responsabilidad con el cuidado ambiental y de la extracción de los recursos se debe invertir en seguridad ambiental para precautelar la biodiversidad y el desarrollo social.

En tal sentido, la corriente conservacionista, está dirigida a la conservación de recursos naturales y la gestora del cuidado ambiental y la que se preocupa de la educación comunitaria para elevar la conciencia ecológica para desarrollar comportamientos favorables de manera equitativa en el área social. La corriente promociona la conservación de los recursos sobre todo en espacios donde estos son escasos. Se desarrolla a través de proyectos que gestionan acciones para la generación de comportamientos que contribuyan a la equidad social.

\section{c.- La corriente resolutiva}

Se puede considerar que esta corriente apoya a la naturaleza que este, sobre todo, sea el centro donde se pueda atender los problemas ambientales por ser la generadora de los recursos y el desarrollo social, además se pueda anexar a la educación ambiental para elevar el nivel de conciencia de las personas.

En este sentido, los encuentros a nivel mundial son donde se toman las decisiones para generar las políticas, los pensamientos y corrientes que asuman la responsabilidad de crear un marco conceptual teórico para poder difundir y se conviertan en generadores de conocimientos y sean transmitidos a través del sistema educativo para un cambio de actitud colectivo. 


\section{d.- Corriente Sistémica}

Para quienes se inscriben en esta corriente, el enfoque sistémico permite conocer y comprender adecuadamente las realidades y las problemáticas ambientales. El análisis sistémico permite identificar los diferentes componentes de un sistema ambiental y de poner en relieve las relaciones entre sus componentes, entre las cuales las relaciones entre los elementos biofísicos y los elementos sociales de una situación ambiental. Según Martínez (2012), el estudio de un medio ambiente dado lleva, en primera fase, a la identificación de los elementos del sistema como los actores, factores y humanos aparentemente responsables de un estado.

Aquí se puede asimilar sobre los beneficios que brinda el medio natural a sus componentes que se sirven de sus bondades ambientales, de sus recursos para desarrollar de manera equitativa igualitaria, sostenible y con equidad.

\section{e.- Corriente científica}

Las proposiciones de la corriente científica integran a menudo el enfoque sistémico y un proceso de resolución de problemas, encontrándose así con las otras dos corrientes anteriormente presentadas. Al igual que en la corriente sistémica, el enfoque es sobre todo cognitivo: el medio ambiente es objeto de conocimiento para elegir una solución o acción apropiada y las habilidades ligadas a la observación y a la experimentación son particularmente requeridas.

En esta corriente, la educación ambiental está a menudo asociada al desarrollo de conocimientos y de habilidades relativas a las ciencias del medio ambiente, campo de investigación esencialmente interdisciplinario, hacía la transdisciplinariedad según manifiesta Sauvé (2010). Se puede deducir que la corriente científica asume los problemas ambientales y busca solucionar con conocimientos para que las personas asuman con responsabilidad utilizando habilidades cognitivas con técnicas investigativas que son requeridas para buscar estrategias que vinculen con la educación ambiental.

\section{f.- Corriente humanista}

Esta corriente, según Sauvé (2005), pone énfasis en la dimensión humana del medio ambiente, construido en el cruce entre naturaleza y cultura. Plantea que el ambiente no es solamente aprehendido como un conjunto de elementos biofísicos que basta con 
abordarlos con objetividad y rigor para comprender, para poder interactuar mejor; por el contrario, corresponde a un medio de vida, con sus dimensiones históricas, culturales, políticas, económicas, estéticas.

Según los autores, conocer el medio ambiente permite relacionarse mejor, y permite estar en condiciones de intervenir en el medio ambiente que facilita el desarrollo humano relacionados con la realidad local con su estilo de vida, aspiraciones de surgir en el medio social como medio de medio de convivencia social, que busca la realidad del ambiente un proyecto de vida

Como se observa, la corriente humanista tiene como prioridad la actividad humana en el medio ambiente, con relación directa entre naturaleza y cultura que se relacionan históricos, política y económicamente como un patrimonio, que se convierten en aliados entre la creación humana y los materiales de la naturaleza.

\section{g.- Corriente Moral /Ética}

Diversas proposiciones de educación ambiental ponen énfasis en el desarrollo de los valores ambientales. Algunos, como lo plantea Reyes (2010), invitan a la adopción de una moral ambiental, prescribiendo un código de comportamientos socialmente deseables, pero más fundamentalmente aun, puede tratarse de desarrollar una verdadera competencia ética, y de construir su propio sistema de valores. No solamente es necesario saber analizar los valores de los protagonistas de una situación, sino que, antes que nada, clarificar sus propios valores, en relación con su propio actuar.

Se considera a la EA como una ética de la vida en medio del auge dominante de economía de la modernidad, es un espacio para formar futuros líderes formados con valores para defender la integridad de la humanidad con equidad a una actividad formadora de responsabilidades, encaminados a reformas sociales, con el uso de mecanismos que contribuyan a la predicción de problemas en su contexto de acción. Según el artículo escrito por González y Figueroa (2009), manifiestan querer poner el acento en los procesos educativos vinculados a los valores, vinculados al medio ambiente, los cuales no acaban de ser incorporados plenamente en los procesos educativos formales con toda su complejidad y amplitud a la vida silvestre sin perturbar. 


\section{Corrientes contemporáneas}

Estas corrientes surgen de las necesidades y requerimientos actuales y dentro de ellas se tienen: h) corriente holística, i) corriente bio-regionalista, j) corriente práxica, k) corriente crítica, 1) corriente etnográfica, m) corriente de la eco-educación, y n) corriente de la sostenibilidad/sustentabilidad.

\section{h.- Corriente Holística}

La corriente holística difiere de las otras corrientes; sus proposiciones están orientadas en reocupaciones de tipo psico-pedagógico relacionado con su medio ambiente de manera participativa desde sus costumbres en un entorno natural, en relación en sus contextos y realidades, que permitan llevar a la reflexionar acerca de los problemas que hoy en día se viven, más bien se trata de una visión muy amplia del mundo con relaciones complejas que no son comprendidas en su totalidad.

\section{i.- Corriente Bio-Regionalista}

Esta corriente motiva a las personas para poner su mirada para volver a los campos, después de la fallida industrialización y de la urbanización masiva que destruyó la integridad social y arraigo la pobreza, destruyó el entorno natural y su biodiversidad, es un movimiento socio-ecológico que se interesa en particular en la dimensión económica de la gestión de este hogar de vida compartida que es el ambiente.

Como se puede inferir, esta corriente no está de acuerdo con el desarrollo industrial, debido al desplazamiento del campesinado a la zona urbana, las promesas de mejorar la calidad de vida con la industrialización solo quedan en una mala experiencia y se desarrolló una tecnología desbastadora del entorno natural.

\section{j.- Corriente práxica}

Esta corriente pone énfasis en el aprendizaje en la acción, por la acción y para mejorar esta última. Según Sauvé (2005), no se trata de desarrollar a priori los conocimientos y las habilidades en vista de una eventual acción, sino de ponerse inmediatamente en situación de acción y de aprender a través del proyecto por y para ese proyecto. El aprendizaje invita a la reflexión en la acción, en el proyecto en curso. 
Lo manifestado por Sauvé nos invita que debemos accionar con propuestas claras, que resuelvan los problemas ambientales con proyectos que se plasmen los logros con resultados favorables que involucren al desarrollo social y al cuidado ambiental con acciones prácticas que conlleven a compromisos responsables dejando las promesas liricas que solo quedad en buenas intenciones.

\section{k.- Corriente de crítica}

La corriente Práxica y la crítica son comúnmente asociadas por tener un fuerte componente humano, en la primera se pone énfasis en el aprendizaje en la acción, por la acción y para mejorar esta última, en la segunda Se trata de una postura valiente, porque ella comienza primero por confrontarse a sí misma y que ella implica el cuestionamiento de los lugares comunes y de las corrientes dominantes (Sauvé, 2005), estas corrientes podrían ser muy relevantes en la actual situación donde existe una gran cantidad de actividades y procesos que se hacen llamar de EA, la corriente crítica entonces entraría a cuestionarse si esas actividades tienen un verdadero impacto, si los postulados y las acciones son congruentes, si se cumplen los propósitos nombrados, así se podría tener mejores procesos o al menos unos que realmente aporten en los diversos contextos.

Un camino a seguir lo que hace esta corriente, hacer una crítica a sus aciertos y errores para continuar con sus objetivos por cumplir organizando acciones que vayan en beneficio de la humanidad y del entorno natural; en este sentido esta corriente insiste, esencialmente, en el análisis de las dinámicas sociales que se encuentran en la base de las realidades con problemas ambientales: análisis de intenciones, de posiciones, de argumentos, de valores explícitos e implícitos, de decisiones y de acciones de los diferentes protagonistas de una situación, cuyo lazo con las problemáticas ambientales debe ser explícito: la educación es a la vez el reflejo de la dinámica social y logros que se espera alcanzar de manera ética con responsabilidad.

\section{l.- Corriente etnográfica}

La propuesta de corriente etnográfica encuentra grandes similitudes con otras como la humanista y la moral, ya que trata de tener en cuenta a las personas en su relación con el entorno, sin embargo, también se propone la observación de otros grupos humanos, y el estudio y aprehensión de las maneras como esos otros grupos humanos tienen una mejor relación con su entorno. 
Galvani, (2001) citado por Sauvé (2005) plantea que la corriente etnográfica pone énfasis en el carácter cultural de la relación con el medio ambiente. La educación ambiental no debe imponer una visión del mundo; hay que tener en cuenta la cultura de referencia de las poblaciones o de las comunidades implicadas.

Lo que podemos rescatar de esta corriente es de gran importancia, promueve a los grupos humanos que están en contacto directo con la naturaleza poniendo énfasis su cultura, desde este punto de vista podemos manifestar que los asentamientos humanos, muchos de ellos tienen un apego amigable con la naturaleza de manera local y que pueden transmitir enseñanzas valiosas sobre cómo proteger el medio ambiente, muchos de estos grupos humanos veneran con respeto a su medio natural porque consideran que les genera el sustento y seguridad en sus vidas.

\section{m.- Corriente de la eco-educación}

Esta corriente está dominada por la perspectiva educacional del medio ambiental que aprovecha la relación con el medio ambiente como determinante en el desarrollo personal, de manera responsable.

Sauvé (2005), distingue dos proposiciones, muy cercanas entre las dos, pero sin embargo distintas, sobre todo en lo relativo a sus respectivos marcos de referencia: la eco formación que se interesa en la formación personal que cada uno recibe de su medio ambiente físico y la eco ontogénesis que pone también en evidencia que las relaciones con el medio ambiente juegan un papel importante en el desarrollo del sujeto, en su ontogénesis.

Según el autor, manifiesta que el medio ambiente influye en el desarrollo en su ontogénesis, que continuara en el futuro en la formación de las personas con las mismas oportunidades estos lazos con el medio ambiente lo que hay que considerar también en educación ambiental como un elemento central y primordial de la ontogénesis.

\section{n.- Corriente de la sostenibilidad / sustentabilidad}

La sustentabilidad se fundamenta en dar apertura al desarrollo social desde las prácticas que sostienen desde la voluntad política, e ideologías que reconocen en la naturaleza su valor intrínseco y respetan las interrelaciones que en ella se llevan a cabo. 
Para Quintero, Fonseca y Garrido (2008), el planteamiento anterior, corresponde a una racionalidad ambiental que difiere de la racionalidad económica cuya base se fundamenta en la gestión de los servicios ambientales que solo usufructúan la naturaleza para satisfacer las necesidades de la especie humana como especie dominante.

Es importante destacar lo que manifiesta estos pensadores, los recursos naturales han generado riquezas enormes beneficiando a unos pocos y dejando en la pobreza a la mayoría de la población, extraen los recursos naturales y en el proceso de la industrialización es contaminación a gran escala al medio ambiente; la naturaleza les brinda sus recursos pero la humanidad no se preocupa en devolver su biodiversidad en su estado natural, a tal punto que estamos al borde de una crisis ambiental que puede ser desbastadora para todas las especies vivientes que está poblando el planeta tierra.

\section{Orientación metodológica}

La metodología seguida para el desarrollo de la investigación tuvo un enfoque cualitativo puesto que se fundamenta en una perspectiva interpretativa centrada en el entendimiento del significado de las acciones de seres vivos, sobre todo de los humanos y sus instituciones (busca interpretar lo que va captando activamente). De acuerdo a Hernández, Fernández y Baptista, (2014), se tipifica como descriptivo, pues ofrece una caracterización de las corrientes en Educación ambiental. Tal y como lo afirma Arias, una investigación descriptiva consiste en la "caracterización de un hecho, fenómeno, individuo o grupo, con el fin de establecer su estructura o comportamiento" (2012.p.24). Los resultados de este tipo de investigación se ubican en un nivel intermedio en cuanto a la profundidad de los conocimientos se refiere.

Para cumplir con el objetivo de esta investigación se utilizaron los métodos teóricos y empíricos que de acuerdo con Salgado y Quintero (2015) tiene su fundamento en la experimentación y la lógica empírica, que, con la observación de fenómenos y su análisis estadístico, es el más usado en el campo de las ciencias sociales y en las ciencias naturales. El método inductivo-deductivo permitió obtener una conclusión general partiendo de primicias con datos particulares que se presentaron al momento de considerar la aplicabilidad de varias corrientes teóricas en educación ambiental con especial atención en el manejo de los recursos naturales del Ecuador. El método analítico- 
sintético permitió determinar los elementos y características de cada corriente, sus ventajas y desventajas.

Se analizaron los criterios de los investigadores, expertos en la Educación Ambiental, declaraciones de la ONU, documentos importantes sobre la educación ambiental.

\section{Resultados}

La educación ambiental está limitada a un enfoque naturalista y no integra las preocupaciones sociales en el tratamiento de las problemáticas ambientales. La educación para el desarrollo sostenible permitiría paliar esta carencia. Debe orientar como medio de prevención a los problemas de carácter ambiental, con la intención de alcanzar el desarrollo sostenible, lo que permitirá a la comunidad el desenvolvimiento de sus potencialidades biofísico y cultural que asegure su permanencia a través del tiempo. Las corrientes teóricas en educación ambiental facilitan el conocimiento y los principios del cuidado del ambiente y aportan la fundamentación suficiente para desarrollar habilidades en el manejo del ambiente, permitiendo a la sociedad relacionarse con su entorno natural.

Analizar las diferentes corrientes ambientalistas, supone cambiar los modelos de pensamiento de los estudiantes y de la comunidad, reorientar la práctica profesional y humana, de manera que crea ajustes en la manera de entender el mundo y sobre la manera de actuar que hoy exhibe la humanidad. Este aporte permitirá llevar a la práctica los conocimientos relacionados con el ambiente y asegurar una educación ambiental sostenible con responsabilidad, elevando el nivel de conciencia de las personas sobre la protección del medio ambiente.

\section{Conclusiones}

El conocimiento veraz y pertinente sobre las corrientes de pensamiento en la educación ambiental, junto a la comprensión del medio ambiente, su comportamiento, manejo adecuado y racional, permite la participación responsable y eficaz en la prevención y solución de los problemas ambientales por parte de la comunidad, contribuyendo en la mejora de la calidad del entorno natural. La educación debe ser observada como una herramienta fundamental en la formación de ciudadanos amigables con el medio ambiente y la perspectiva resaltada de la EA incluye elementos significativos como la paz, la violencia, la democracia y la pobreza, los cuales son 


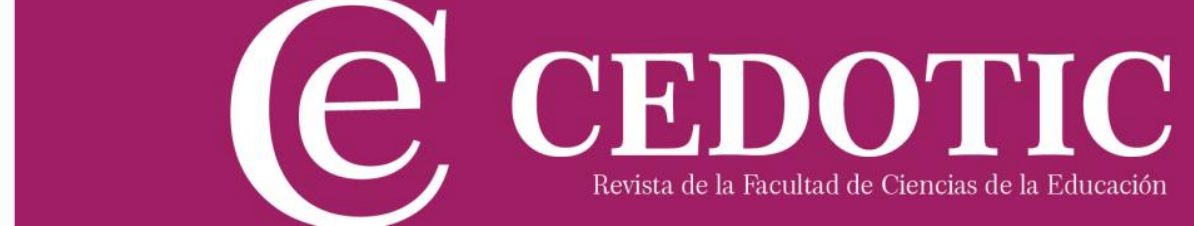

fundamentales desde el punto de vista pedagógico. Su fundamento ya se encuentra en el pensamiento crítico de Freire o en el enfoque cognitivo de Gardner (2008) para quien es vital en la actualidad y en el futuro desarrollar cinco tipos de mentes, entre esas, la mente respetuosa y la mente ética, las cuales permiten construcciones sociales basadas en el respeto al otro y la unificación de valores que propenden por ambientes más sanos.

En este sentido, la EA debe dirigirse hacia la comprensión de la realidad del sujeto y al despertar de la conciencia del mismo que le permita identificarse como ser posibilitador de cambios. Dicho proceso educativo es clave en la formación de convicciones y actitudes éticas, económicas y estéticas, que constituyen la base de una disciplina hacia el medio ambiente, garantizando su protección, mantenimiento y preservación. En la medida en que la educación sea modificada, se podrá alcanzar transformaciones significativas en todos los campos y de ahí que la apuesta para darle un nuevo significado al medio ambiente dependa, esencialmente, del sentido y el papel que se le imprima a la educación.

A medida que los foros internacionales han venido promoviendo la construcción de modelos de enseñanza en educación ambiental, es muy poco lo que se ha logrado a través de los acuerdos internacionales, proyectos y campañas de protección ambiental ya que los países industrializados no han cumplido con los desembolsos de los recursos económicos para asegurar los cambios económicos y políticos que la región y el mundo amerita. Estas reflexiones de pensadores y corrientes ambientalistas permiten reorientar y evaluar los programas educativos en el Ecuador en función de un enfoque social del ambiente. 


\section{Referencias bibliográficas.}

Arias, F (2012). El proyecto de investigación. Editorial Episteme. Caracas. Venezuela

Avendaño, W (2012). La Educación Ambiental (EA) como herramienta de la responsabilidad social (rs). Revista Luna Azul. 35: 94-115. Universidad de Caldas.

Carta de Belgrado. Seminario de Educación Ambiental, 13-22 de octubre de 1975.

Copello, M., Nunes, M., Langoni, M. ; Russo, D. (2008). Algunas propuestas de educación ambiental en la escuela. Parte II: Uso responsable del agua: ¿Es un problema? ¿Yo tengo algo que ver con él? Quehacer Educativo, abril, 44-51.

Corcuera, P; Ponce de León G, L. (2004). Tendencias de los movimientos conservacionistas y el surgimiento de la Eco-Ética Sociológica. vol. 19, núm. 56, septiembre-diciembre, pp. 199-211 Universidad Autónoma Metropolitana Distrito Federal, México

Cugler, A. (2010). ¿Se puede aplicar la educación ambiental en la actividad turística? Ponencia presentada en la Conferencia Turismo y Educación Ambiental, julio, Lima, Perú.

Cumbre Mundial sobre el Desarrollo Sostenible (2002), 26 de agosto al 4 de septiembre, Johannesburgo, Sudáfrica.

Declaración de Río sobre el Medio Ambiente y el Desarrollo, 14 de junio de 1992, Río de Janeiro, Brasil.

Gardner, H. (2008). Las cinco mentes del Futuro. Bogotá: Paidós 
González G, E. (2006). Campo de partida. Educación ambiental y educación para el desarrollo sustentable: ¿tensión o transición? Trayectorias, vol. VIII, núm. 20-21;5262 Universidad Autónoma de Nuevo León, Monterrey, Nuevo León, México

González G, E; Figueroa de K, L. (2009). Los valores ambientales en los procesos educativos: realidades y desafíos. REICE: Revista Electrónica Iberoamericana sobre Calidad, Eficacia y Cambio en Educación 7.2: 1-21

González, E.; Valdez, R. (2012). Enfoques y sujetos en los estudios sobre representaciones sociales de medio ambiente en tres países de Iberoamérica. Revista de Investigación Educativa, 14.

Gutiérrez, J.; Pozo, T. (2006). Modelos teóricos contemporáneos y marcos de fundamentación de la educación ambiental para el desarrollo sostenible. Revista Iberoamericana de Educación, 41, 21-68.

Hernández, R, Fernández, C y Baptista, L (2014). Metodología de la Investigación. Editorial Mac Graw Hill. México.

Martínez C, R. (2012). Ensayo crítico sobre educación ambiental. Revista Electrónica Diálogos Educativos. Nº 24, Vol. 12.

Organización de las Naciones Unidas (ONU). (1972). Declaración de Estocolmo sobre el Medio Ambiente Humano. Estocolmo, 5-16 de junio.

Quintero S, M; Fonseca H, C; Garrido S, F. (2008). Revisión de las corrientes teóricas sobre el medio ambiente y los recursos naturales. Revista Digital Universitaria. Volumen 9 Número 3.

Reyes, J. (2010)."Educación ambiental: rumor de claroscuros". Publicada en Los Ambientalistas, revista de Educación Ambiental. Septiembre-diciembre

Salgado, E y Quintero, H (2015). La estrategia didáctica en el aprendizaje de las fracciones. Universidad del Zulia. 


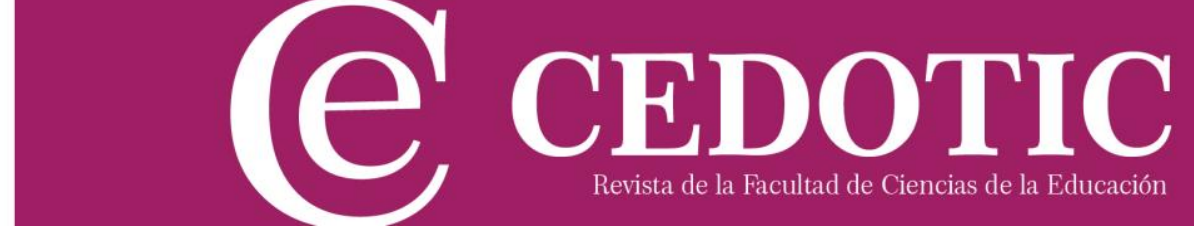

Sánchez T, D; Aguilera P, M. (2014). Corrientes del ambientalismo y alternativas de gestión desde la sustentabilidad y la ética ambiental. Semestre Económico, volumen 17, No. 35, pp. 149-160. Medellín, Colombia.

Sauvé, L. (2005). Una cartografía de corrientes en educación ambiental. En Sato, M. \& Carvalho, I. (Orgs.). A pesquisa em educação ambiental: cartografías de uma identidadde narrativa em formação. Porto Alegre: Artmed.

Sauvé, L. (2010). Educación científica y Educación Ambiental: un cruce fecundo. Enseñanza de las Ciencias, 28(1), 005-018. Disponible en https://www.raco.cat/index.php/Ensenanza/article/view/189092/353371. Consultado el 8 de noviembre 2018 